\title{
Authoring Consistent Landscapes with Flora and Fauna
}

\author{
PIERRE ECORMIER-NOCCA, LIX, Ecole Polytechnique/CNRS, Institut Polytechnique de Paris, France \\ GUILLAUME CORDONNIER, ETH Zürich, Switzerland and Inria, Université Côte d'Azur, France \\ PHILIPPE CARREZ, Immersion Tools, France \\ ANNE-MARIE MOIGNE, Muséum national d'Histoire naturelle - UMR 7194, HnHp, MNHN, UPVD, CNRS, France \\ POORAN MEMARI, LIX, CNRS/Ecole Polytechnique, Institut Polytechnique de Paris, France \\ BEDRICH BENES, Purdue University, USA \\ MARIE-PAULE CANI, LIX, Ecole Polytechnique/CNRS, Institut Polytechnique de Paris, France
}

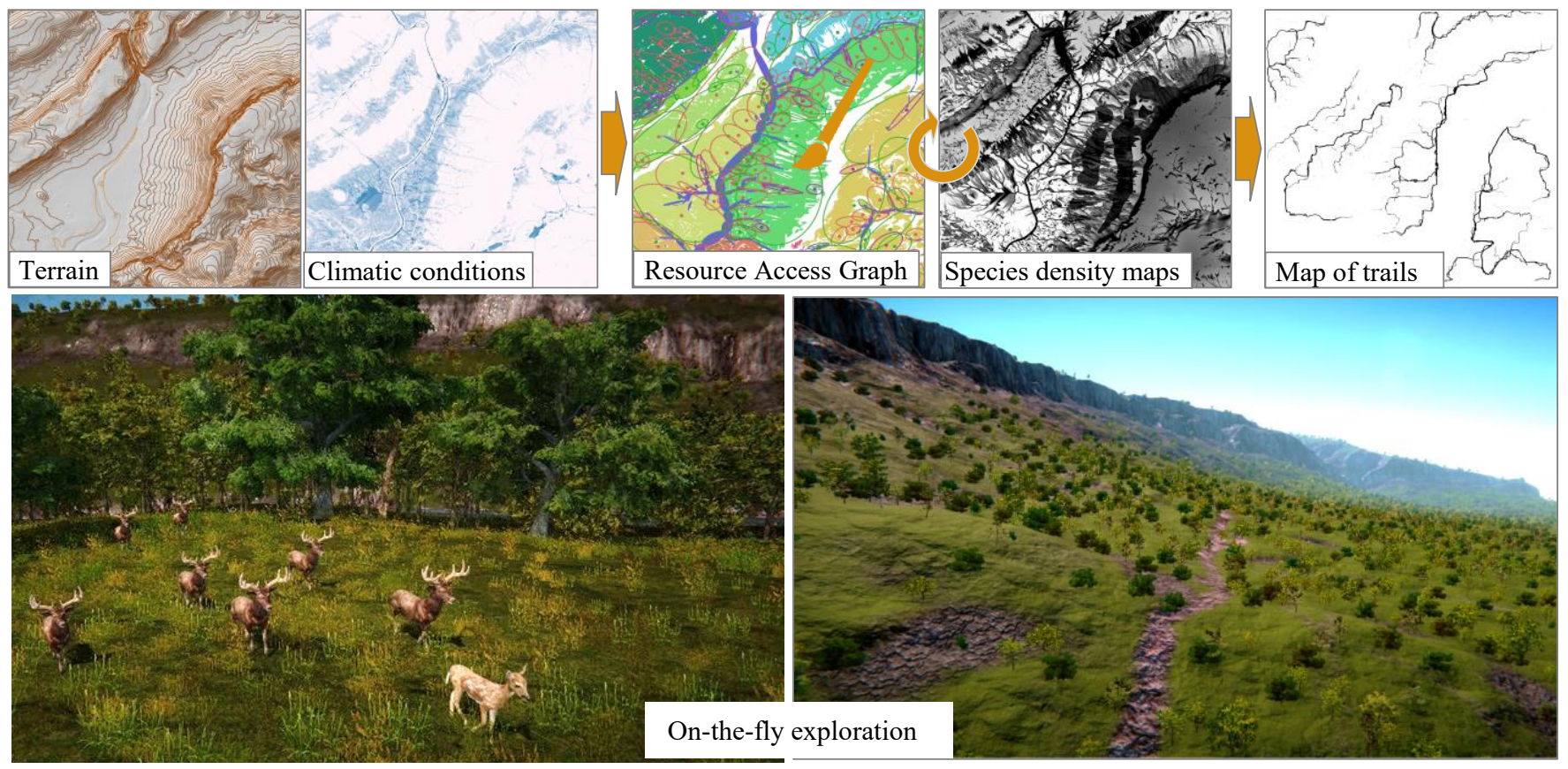

Fig. 1. From an input terrain, climatic conditions desired proportions between competing species and their dependency through the food-chain, our algorithm iterates on a resource access graph to compute species density maps, enabling us to extract animals' daily paths. The resulting, consistent 3D landscape can be explored on the fly, showing vegetation, animals, and the trails they generate. Maps can be edited at any stage using painting tools.

We present a novel method for authoring landscapes with flora and fauna while considering their mutual interactions. Our algorithm outputs a steadystate ecosystem in the form of density maps for each species, their daily

Authors' addresses: Pierre Ecormier-Nocca, LIX, Ecole Polytechnique/CNRS, Institut Polytechnique de Paris, Palaiseau, France, pierre.ecormier@polytechnique.edu; Guillaume Cordonnier, ETH Zürich, Zürich, Switzerland, Inria, Université Côte d'Azur, Sophia Antipolis, France, guillaume.cordonnier@inria.fr; Philippe Carrez, Immersion Tools, Saint-Brieuc, France, philippe.carrez@immersion.tools; Anne-Marie Moigne, Muséum national d'Histoire naturelle - UMR 7194, HnHp, MNHN, UPVD CNRS, Paris, France, anne-marie.moigne@cerptautavel.com; Pooran Memari, LIX CNRS/Ecole Polytechnique, Institut Polytechnique de Paris, Palaiseau, France, memari@ lix.polytechnique.fr; Bedrich Benes, Purdue University, West Lafayette, USA, bbenes@ purdue.edu; Marie-Paule Cani, LIX, Ecole Polytechnique/CNRS, Institut Polytechnique de Paris, Palaiseau, France, marie-paule.cani@polytechnique.edu.

(C) 2021 Copyright held by the owner/author(s). Publication rights licensed to ACM. This is the author's version of the work. It is posted here for your personal use. Not for redistribution. The definitive Version of Record was published in ACM Transactions on Graphics, https://doi.org/10.1145/3450626.3459952. circuits, and a modified terrain with eroded trails from a terrain, climatic conditions, and species with related biological information. We introduce the Resource Access Graph, a new data structure that encodes both interactions between food chain levels and animals traveling between resources over the terrain. A novel competition algorithm operating on this data progressively computes a steady-state solution up the food chain, from plants to carnivores. The user can explore the resulting landscape, where plants and animals are instantiated on the fly, and interactively edit it by over-painting the maps. Our results show that our system enables the authoring of consistent landscapes where the impact of wildlife is visible through animated animals, clearings in the vegetation, and eroded trails. We provide quantitative validation with existing ecosystems and a user-study with expert paleontologist end-users, showing that our system enables them to author and compare different ecosystems illustrating climate changes over the same terrain while enabling relevant visual immersion into consistent landscapes.

CCS Concepts: • Computing methodologies $\rightarrow$ Procedural animation Additional Key Words and Phrases: Natural Phenomena, Ecosystems 


\section{ACM Reference Format:}

Pierre Ecormier-Nocca, Guillaume Cordonnier, Philippe Carrez, Anne-Marie Moigne, Pooran Memari, Bedrich Benes, and Marie-Paule Cani. 2021. Authoring Consistent Landscapes with Flora and Fauna. ACM Trans. Graph. 40, 4, Article 105 (August 2021), 13 pages. https://doi.org/10.1145/3450626.3459952

\section{INTRODUCTION}

Beautiful landscapes result from the interaction between various phenomena, such as climate, erosion, vegetation, and animals. While these phenomena have a strong influence on landscape appearance, their combined effects are not always well-understood. Only some of them, namely climate, erosion, and plant ecosystems, were simulated and combined so far. Animated fauna, essential for generating lively landscape in movies, simulators, or games, is typically added manually in the last stage, requiring a considerable amount of user effort.

Our first key observation is that the presence of animals has a critical visual impact on landscapes, even when they are out of sight Indeed, they shape clearings and trails through the vegetation while compete for space and resources, which, in turn, affects erosion. Therefore, the standard pipeline, where animals are added on top of a terrain covered with plants, while neglecting their interactions should be revisited.

The second key observation is that modeling a landscape where various life forms co-exist can be done consistently without a full simulation, and while enabling intuitive user control. Designers of virtual environments aim at immersing the audience in a specific ecosystem, with either real or imaginary sets of plants and animal species, in given proportions. Similarly, immersion in a 3D ecosystem that matches observed data and current hypotheses would be a plus for experts such as biologists or paleontologists as a new way to explore their models. While a full, simultaneous simulation of terrain formation, vegetation, and wild-life competition would be challenging due to the multiple time scales involved, it would also fail to match these authoring needs, with no guarantee on the set of surviving species and their proportions.

Our solution increases the quality of CG landscapes by generating coherent, yet controlled ecosystems with flora and fauna, interacting with the terrain. It considers plants and animals as different food chain levels, which are iteratively instantiated from lower to upper levels. Our method allows both intuitive authoring and the onthe-fly exploration of the resulting landscape, with consistently positioned plants and animated animals along relevant daily paths.

In a typical use-case, the user specifies climatic conditions over an input terrain and a set of species of plants, herbivores, and carnivores with optional, desired proportions after the competition. The resulting ecosystem is then computed in the form of a set of editable density maps for each species over the terrain. At each level of the food chain, the impact of the generated individuals is propagated back to the resources they consume. The paths between them are computed, enabling to account for grazing, foraging, and erosion along the main trails. The resulting landscape, including both flora and animated fauna, is finally consistently instantiated to allow interactive exploration. Fig. 1 shows an example of such interactive authoring and exploration of a populated landscape.
We achieve these results thanks to several key choices and approximations: First, in addition to the choice of species at each food-chain level, we provide users with direct control over their proportions. We compute the actual number of specimens and their distribution over the terrain from this input, thanks to a new procedural competition algorithm ruled by the simplifying hypothesis that the resulting ecosystem should be at a steady-state. Second, this algorithm builds on a novel hierarchical data-structure, called the Resource Access Graph (RAG), which embeds resources for each species with their location and accessibility as a layered graph, enabling efficient, yet consistent species instantiation over the terrain.

We claim the following contributions: 1) we introduce the RAG, a hierarchical, biology-driven, embedded graph of resources, which encodes local interactions among food chain levels and enables us to model paths between resources; 2) we introduce a procedural competition algorithm to build a fast approximation of a steadystate ecosystem at each level of the food chain; 3) we propose a method for the consistent, on-the-fly instantiation of plants and animals during interactive landscape exploration.

\section{RELATED WORK}

We do not focus on terrain generation, and we refer the reader to the surveys [Galin et al. 2019; Smelik et al. 2014].

Plant ecosystems have been addressed mostly by the simulation of competition for resources. Deussen et al. [1998] first used plant competition to simulate plant distributions, a work recently extended to layered ecosystems [Makowski et al. 2019]. Various approaches attempted to combine ecosystems with other phenomena: Benes et al. [2011] modeled the interaction of urban layouts with plant competition for resources while considering human urban management, Gain et al. [2017] simulated and learned plant distributions and used them as interactive brushes over a terrain, Cordonnier et al. [2017] modeled plant interaction with terrain erosion, while [Kapp et al. 2020] trained a deep neural network to learn and generate consistent vegetation over an input terrain. In contrast with our work, only interaction between flora and terrain was considered, neglecting the impact of animals on both vegetation and erosion. Moreover, although we build on concepts from simulation methods such as fitness [Alsweis and Deussen 2005; Gain et al. 2017] to give local priority to dominant species, our method focuses on steady-state ecosystems, offering control over species proportions.

Regarding fauna, the seminal works of Reynolds [1987; 1999] introduced boids that simulate the self-synchronized movement of a flock of birds. Models capable of making more refined decisions based on the velocity of animated agents [Paris et al. 2007; van den Berg et al. 2008] or their synthetic vision [López et al. 2019; Ondřej et al. 2010] have been developed to mimic observed behaviors. Animals have also been studied to increase artistic freedom and control, especially when interacting with the environment. The works [Wang et al. 2014; Xu et al. 2008] allow the user to control the shape taken by a group of animals. At the same time, Ecormier-Nocca et al. [2019] extended control to both the shape and distribution of animals within a herd. However, while these approaches produce visually plausible animations, they do not consider the relationship 


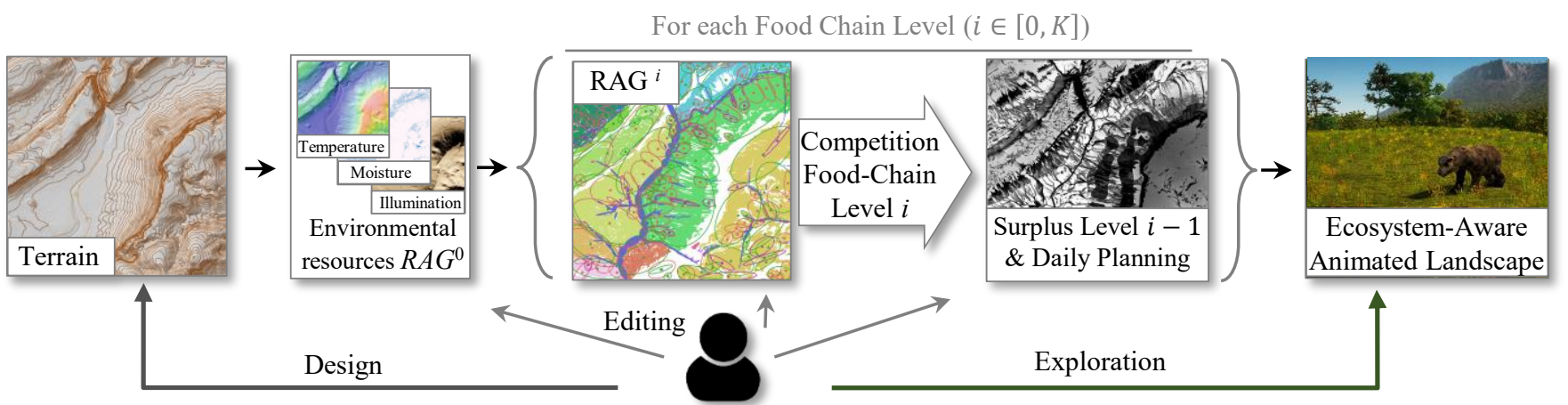

Fig. 2. System overview: After initializing resource maps, we process each food chain level, from plants to carnivores, computing the corresponding level of the Resource Access Graph (RAG), the result of competition between species at this level, animals daily paths, and the remaining surplus of resources from the previous level. The resulting set of density maps, together with a map of eroded trails on the terrain and daily planning for animals, are used to generate the animated, ecosystem-aware-landscape. The user can intuitively edit results by over-painting any of the maps.

with other essential factors such as vegetation, accessibility of the terrain, or scarcity of resources, which is one of this paper's goals.

Competition between species modeled by biologists for both flora and fauna, is expressed as differential equations, leading to predictive, dynamic models for populations' ecological niches, and allowing decision making for global resource management. For instance, motivated by conservation biology for ecology and environmental analysis, [Loreau 2010] demonstrated the impact of biodiversity on ecosystems, and [Shifley et al. 2017] provided a comprehensive survey of dynamic models for forest landscapes. In contrast, we are not introducing a simulation method for dynamic ecosystems. Instead, we focus on an authoring tool to generate a landscape that maintains ecosystem consistency while building on user input either imaginary or extracted from real data. This input flexibility is also why our system does not aim to provide a complete statistical occupancy model or a Bayesian simulation of animal population dynamics. We refer the reader to the book [MacKenzie et al. 2017] for a comprehensive insight into mathematical modeling of ranging patterns and occupancy dynamics.

\section{OVERVIEW}

Our goal is to generate a consistent, animated landscape matching flora and fauna either chosen by design by an artist, observed on the terrain by a biologist, or inferred from remains - such as animal bones and plant pollen - by a paleontologist. To match such authoring needs, we introduce a novel processing pipeline (see Fig. 2) consistently generating and embedding plants, herbivores, and carnivores over a given terrain, under a steady-state hypothesis.

The steady-state hypothesis postulates that the growth of populations in ecosystems is limited by two main factors: natural death (caused by old age, disease, food shortage, climate, etc.) and the action of predators. Without these, the population would grow exponentially every year by a species-dependent factor, called the growth rate, and given by the average yearly production of the species (volume produced by plants, number of newborns for animals) per unit. The yearly growth of a given population is its size multiplied by this rate. The steady-state hypothesis states that yearly deaths precisely compensate for annual growth for each species in the ecosystem. In particular, no more than the yearly growth of a species can be used as a resource for another species. We call surplus the annual amount of such resource finally unused and supposed to naturally die over the year.

\subsection{Knowledge, Input and, Output}

Biological knowledge about living species embedded in our system was extracted from a plant database [Tela-Botanica DB] and an animal species database [Animal-Diversity-Web DB]. It includes the set of yearly resources required for each species (minerals, light, and water for plants, water, and food for animals), the minimal and maximal temperature a plant can bear, the average motion speed of animals, and their ability to climb slopes and cross rivers.

Knowledge about the set of necessary resources for species is used to classify them into food-chain levels, where species from a given level feed on the previous level and serve themselves as resources for the next level. In our implementation, we only consider vegetation, herbivores, and carnivores as distinct food-chain levels, but generalizing our method to more levels would be straightforward. Extension to complex ecosystems with food-graphs instead of linear food-chains is discussed in Section 7.

User input includes a digital elevation model describing the targeted terrain, together with all the necessary information to compute local environmental resources for plants, namely a minerals map, the terrain orientation, latitude, altitude, and the extreme temperatures at sea level - describing the targeted climate. We adapted the method from [Gain et al. 2017] to extract yearly moisture, extreme temperatures, and sun-light maps over the terrain from this input. The user may alternatively directly provide the maps. The user also lists the set of species they would like to see in their ecosystem, together with the optional, desired proportions between species at the same food-chain level.

The output is a set of maps representing the density of presence of each species over the terrain, and a map of eroded trails. In contrast with the maps used for plants, we use piece-wise constant maps for 
animals to represent their ability to move anywhere within speciesspecific confinement regions, delimited by natural frontiers such as rivers or cliffs that the animals cannot cross. Since herds have non-uniform probabilities of presence over these regions depending on the location of resources, we also compute daily itineraries to model their typical routes. This output is finally used to generate an animated landscape populated with plants and animals of various species, with clearly distinguishable trails.

\subsection{Concept of Resource Access Graph}

Key to our solution is a novel data structure, a hierarchical directed graph called the Resource Access Graph (RAG). It encodes the interactions between species at different levels of the food chain and their embedding onto the terrain. See Fig. 3.

At each level $i$ of the hierarchy, RAG nodes are the individual resources available for the set of species at level $i$ of the food chain, together with their embedding on the terrain. To match the steadystate hypothesis, resources are set to surplus from the previous food chain level, representing the amount that can be consumed with no impact on the ecosystem equilibrium. The embedding of a resource node is the region of the map where it can be found, e.g., the region covered by a grass meadow, or the confinement region between a river and a cliff, where a herd of herbivores grazes. As the name "resource access graph" indicates, RAG edges - labeled by indicators of species - encode the ability of a given species to travel between resource nodes and are labeled with the average traveling time. Note that we use an oriented graph for the RAG because the abilities and traveling times up or down a slope are different.

The RAG hierarchy expresses that each species may be a resource for other species, and should thus be encoded as a higher-level resource node; e.g., antelopes confined in a sub-region of the terrain between a large river and a cliff are food resource to wolves, so the strongly connected component of the RAG they travel in to graze, will correspond to an individual resource node at the next level of the RAG, granted that these antelopes produced some surplus.

\subsection{Processing Pipeline}

The processing pipeline (Fig. 2) consists of progressively computing the RAG from the bottom to the top of the food chain. The solution to competition and positioning of species at the previous foodchain level is used while calculating the next one. Density maps and traveling edges in the RAG are then used to generate the output.

More precisely, the first level of the RAG, $R A G^{0}$ (i.e., environmental resources for plants), is initialized by considering each map's cell as an individual node. Then, for each level $i$ up the food chain:

(1) The associated level of the RAG, encoding resources for species, their location over the terrain, and their accessibility, is built;

(2) Competition at food chain level $i$ is solved using a new, procedural method to compute steady-state results that best match the desired proportion of species specified by the user;

(3) Daily paths between resources are generated for each animal species, and surplus maps describing unused resources from the previous level of the food-chain are computed.

Finally, the paths in the RAG are refined, combined and exported as a map of eroded trails over the terrain. The collection of species density-maps and daily paths for animals are processed to generate the ecosystem, where surpluses are displayed in the form of denser or higher plants (depending on the species) or more youths among animal herds, to inform users about the liveliness of the ecosystem.

Users can interactively explore the resulting animated landscape and further tune it by editing density maps from any food-chain level. While increasing the density of species may not match realism due to insufficient resources (a warning is displayed), decreasing them is always possible and will propagate up and down the foodchain for consistency (fewer resources at the next level, more surplus at the previous one). Such editing can be used to model external events such as death due to fire or diseases.

\section{COMPETITION BETWEEN SPECIES}

Species at the same food chain level compete for resources. The second step of the processing pipeline consists of computing a set of density maps describing how species settle in different terrain regions, given the locally available resources for which they compete. To allow authoring, user-defined target proportions between species can be accounted for, although this may result in less populated ecosystems. Without such proportions, species which best fit local resources are favored in each region. In both cases, our solution ensures that all survival conditions are met.

\subsection{Survival Constraints}

Among the information found in environmental and biological databases, some features can be extracted whatever the species (flora or fauna), and their resources (environmental resource, flora or fauna). Our solution is based on the list of features below (see supplemental documents for the values used for each species).

A population unit for a given species is a small group of the specimen that live together. We define it, for flora, as the least dense group of individual plants that may span a single grid-cell of the terrain, and for fauna, as the smallest typical herd, a herd being defined as a group of animals traveling together. Solitary animals (e.g., a bear) are considered as herds of size one.

The consumed resource $c(s, r)$ for a species $s$ and a resource $r$ denotes the amount of $r$ a unit of $s$ typically consumes annually, and which is therefore not available anymore for other species. Note that in the case of temperature, a resource to plants, this amount is set to zero since flora's presence has a negligible effect on temperature.

The temporal variation $\left[r_{\min }, r_{\max }\right]$ of a resource $r$, is the interval between the minimum and maximum amounts of $r$ available over a year (e.g., the interval spanned by temperature, seen as a resource to plants; or the interval between zero and the yearly growth of grass, for a meadow used as a resource for herbivores). Note that for resources with seasonal changes, we use more detailed data if the data is available. For example, we use temporal variations at the scale of a month for temperature and precipitations.

The fitness range, for each species $s$ with respect to a resource $r \in R(s)$ (their set of necessary resources), is the interval $\mathcal{F}(s, r)=$ $\left[\mathcal{F}_{\text {min }}(s, r), \mathcal{F}_{\text {max }}(s, r)\right]$, between minimum and maximum values of $r$ for which the growth of species is not limited by the environment. 

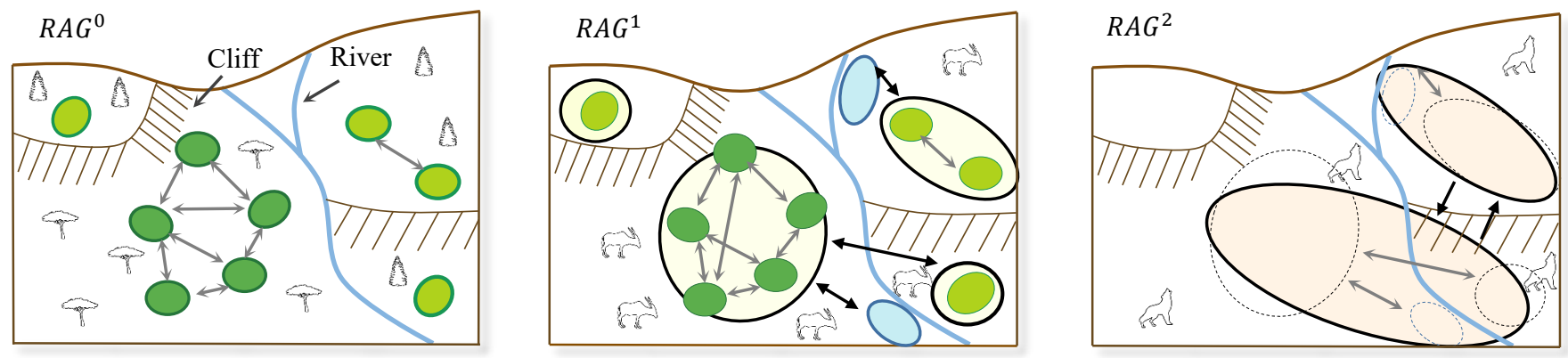

Fig. 3. Concept of Resource Access Graph showing surplus nodes colored per species. $R A G^{0}$ encodes resources for plants. $R A G^{1}$ encodes resources for herbivores such as meadows and river banks. Antelopes can cross the river only at a ford and are trapped by cliffs, leading to three confinement regions. $R A G^{2}$ encodes resources for carnivores, here wolves feeding on antelopes. The connected components of $R A G^{1}$ where antelopes produce surplus (bottom and right ones) are merged as resource nodes in $R A G^{2}$. Edges express the fact that wolves can go up and down cliffs, with different traveling times.

A confinement region for $s$ is a part of the terrain from which specimens cannot exit due to lack of mobility for plants, and to obstacles such as cliffs, rivers, or too dense vegetation for animals.

Given these definitions, any created population unit of a species $s$ should survive on local resources provided by their confinement region. The more yearly variations of local resources fit within the fitness ranges for $s$, the more $s$ will tend to settle in this specific region. Simultaneously, the settlement should not be allowed if these intervals do not overlap. Lastly, population units of $s$ should only be created if a yearly amount $c(s, r)$ of each resource can be reserved for them. Our solution ensures the consistency of the created ecosystems by maintaining these constraints.

\subsection{Local Species's Fitness Score}

Let us consider a region where the set of resources $r \in R$ for species at a given food-chain level can be found. To compute which of these species are the most likely to settle there, we calculate a fitness score fit $(s, R)$ for each of them in the following way.

For each resource $r \in R$, we compute the fraction of the species' fitness range $\mathcal{F}(s, r)$ that lies within the annual variation $r_{\text {year }}$ of the corresponding resource. Since the sparsest resource determines the ability for $s$ to settle there, we use the minimum of these values, leading to:

$\operatorname{fit}(s, R)=\min _{r \in R}\left(\frac{\min \left(\mathcal{F}_{\text {max }}(s, r), r_{\text {max }}\right)-\max \left(\mathcal{F}_{\min }(s, r), r_{\text {min }}\right)}{\left(r_{\text {max }}-r_{\text {min }}\right)}\right)$,

When the monthly temporal variations are provided (e.g., for temperature), we use several terms per resource in the minimum above. This results in a continuous score $0<=\operatorname{fit}(s, R)<=1$, where zero indicates that $s$ cannot survive, while one shows a perfect fit, all resource variations being within the species' fitness ranges)

\subsection{Competition Algorithm}

Let us now consider a terrain divided into a set of confinement regions $C_{j}$, each with its resources $R_{j}$. We denote $q\left(r, C_{j}\right)$ the annual available amount of $r \in R_{j}$ in the region $C_{j}$.

Note that the same set of regions needs to be used for all species. When the traveling abilities are different among species at this level of the food chain (e.g., goat vs. bison), we use the finest partition of the terrain, computed as the intersection of species-specific confinement regions. In this case, the created population units are assigned to the species' original confinement regions.

Competition considering target proportions of species. Starting from zero densities over the terrain, we successively, tentatively, increase by one the number of population units in each species. Our greedy solution only retains the one, among these attempts, that brings the system the closest to the desired proportions. The newly created unit is then added to the best-adapted confinement region $C_{j}$ on the terrain, i.e., considering only the $C_{j}$ with strictly positive fit $\left(s, R_{j}\right)$, we sort them by decreasing values, and use the first one, if any, where $\forall r \in R_{j}, q\left(r, C_{j}\right)>c(s, r)$. If this succeeds, the population unit is created in $C_{j}$, and the amounts $q\left(r, C_{j}\right)$ are decreased by $c(s, r)$, for each resource $r \in R_{j}$. This process is repeated until no new population unit can be created.

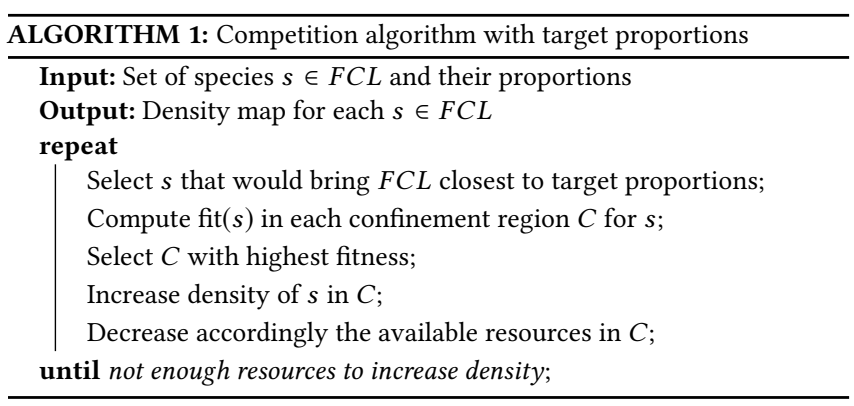

Competition without target proportions. For each confinement region $C_{j}$ : Starting from zero, we iteratively increase the number of population units (and accordingly decrease the amount of available resources), choosing species with highest strictly positive fitness $f i t\left(s, R_{j}\right)$ matching $\forall r \in R_{j}, q\left(r, C_{j}\right)>c(s, r)$. We stop when there are not enough remaining resources in $C_{j}$.

Discussion. Although we reuse at a coarser grain (annually for animals), some of the concepts introduced for plant-ecosystems, such as asymmetric competition and fitness score [Alsweis and Deussen 2005; Gain et al. 2017], our method strongly differs from 


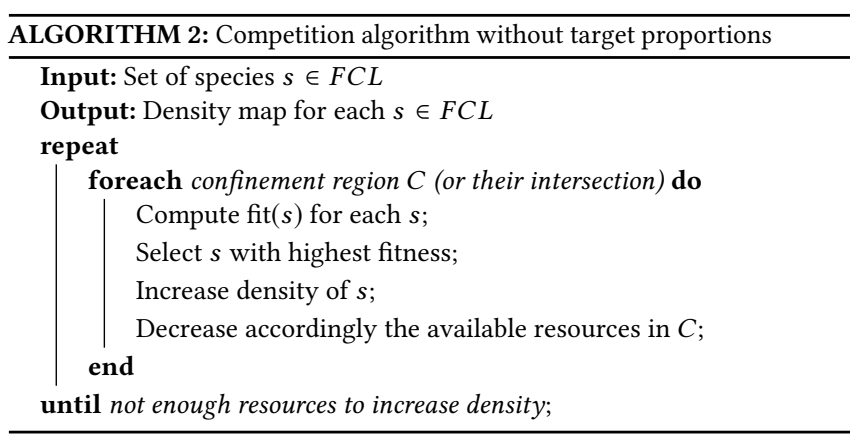

previous work since it does not belong to ecosystem simulation methods. We do not simulate plant seeding, growing, dying, or succession of birth, aging, and death for animals. Our approach focuses on the local competition. Therefore, while we could build on sand-box simulations to input correct proportions between species, a method like Ecobrush [Gain et al. 2017] could benefit from our precise plant placement method to complement their brushes that paint uniform distributions. Even considering vegetation only, this would result in more varied yet consistent landscapes since we position each plant where it is the most likely to develop.

The simple, deterministic algorithm for best matching the target proportions may not provide an exact solution, given that populations are created per units of fixed size. Moreover, with survival constraints being enforced, non-plausible input proportions will result in sparse ecosystems, leaving a large surplus of resources. Yet, this algorithm has the benefits of iteratively decreasing distance to target proportions while maintaining consistency. The second algorithm, which instead uses a region-based strategy, is guaranteed to iteratively reduce the local resource surplus - while again, the latter might not reach zero because of the unit sizes we use. It results in diverse ecosystems, granted that species that fit well there were defined. Creating animals per herds and leaving some surplus among vegetation and lower-level species add to the realism.

Although the algorithms are greedy, our two solutions have the advantage of being efficient to compute given the relatively small, typical number of species at each food-chain level and accounting well for local resources available in each region of the terrain, leading to locally distinct species settlement.

\subsection{Output: Species Density Maps}

After competition is solved, local density for a plant species in each grid cell is set to the proportion of the terrain surface covered by the plant. The density is defined for animals, in each species-specific confinement region, as the number of specimens per square meter Densities are displayed in gray-scale, leading to piece-wise uniform colors for fauna maps (see Fig. 4(c)).

The resulting maps makes the output easy to interpret for the user, who can quickly detect the most populated region in a given map, or the local preeminence of species. This intuitive visualization also makes the maps easily editable using a painting interface.

\section{BOTTOM-UP RAG CALCULATION}

It would not be practical to propagate the different sets of maps up the food-chain directly, to check for types, quantities, and accessibility of resources while considering the species traveling abilities, their need for water, and the fact that they feed on other species. To simplify data computation and make these queries efficient, we extract all the necessary information and represent it hierarchically within a graph structure, the Resource Access Graph (RAG) already introduced in Fig. 3. This section provides a formal definition of the RAG and then explains how this structure is progressively built.

We use the following notations, enabling us to express the whole food chain at once: Species in the ecosystem are denoted as $S=$ $\left\{S_{j}^{i}\right\}_{j=1, \ldots, N_{i}}^{i=0, \ldots, K}$, where $F C L^{i}=\left\{S_{j}^{i}\right\}_{j=1 . . N_{i}}$ is the subset of species at food-chain level $i$. In our implementation, $F C L^{0}$ stands for plants, $F C L^{1}$ for herbivores and $F C L^{2}$ for carnivores. $R^{i}$ stands for the set of resources for $F C L^{i}$. These resources are spatialized: in addition to its nature and the available quantity, $r \in R^{i}$ is also described by the area it spans (e.g., water resources - supposed to be infinite in our implementation - span full river banks from which the animals can drink). The Resource Access Graph (RAG) (see Section 3.2) is built from the following elements:

Resource nodes $v$ are vertices of the RAG. They represent one or several resources spanning the same area. Resource nodes $v$ are positioned on the terrain map at the centroid of the associated resource area. Nodes are sorted by layer, corresponding to the different levels of the food chain. The quantity of resource $q(r, v)$ is stored in the nodes of the data-structure, for each resource $r$ available at $v$.

Spatial edges $e$ are oriented edges connecting two nodes from $R A G^{i}$. They are labeled by species $s \in F C L^{i}$, and model the existence of an available traveling path for $s$ between the two nodes on the terrain. They are valued by the traveling time in each direction.

Hierarchical edges $h$ are inter-level edges that connect a node of $R A G^{i+1}$ (e.g., a herd of antelopes, resource to wolves) to the nodes of $R A G^{i}$ used to build it (i.e., the resources nodes for this specific herd). Such edges enable to recover information about a resource, such as the exact areas and paths where a herd can be found.

\subsection{RAG Initialization: Vegetation and Accessibility Maps}

$R A G^{0}$ models the environmental resources for plants, computed from input soil type and climate on the terrain grid, as shown in Section 3.1. A node is created at the center of each grid cell to store this information about local resources. We use the competition algorithm from Section 4 to compute density maps for $F C L^{0}$ (flora) from $R A G^{0}$. Note that this graph is disconnected because plants are restricted to resources in their grid cells.

Artificial spatial edges labeled per plant species are then added to $R A G^{0}$ to connect neighboring resource nodes where the same species grow. We use this feature so that a contiguous area where a given plant species grow (say, a grass meadow) is grouped within a single node in $R A G^{1}$, modeling resource for herbivores.

Finally, we segment the terrain into confinement regions for the whole fauna. More precisely, we compute accessibility maps for an animal species $s$ as follows: The map is initialized as a regular grid, from which all cells that correspond to an obstacle $s$ cannot 


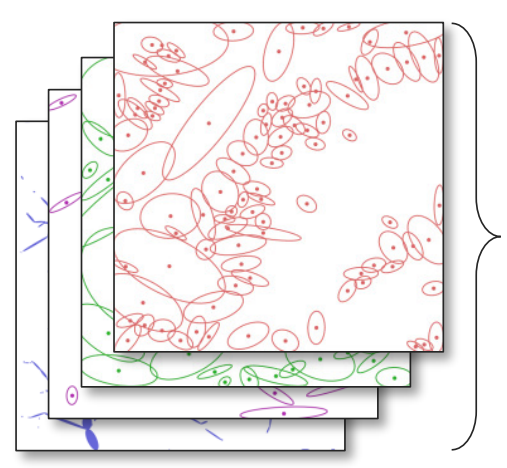

(a) Individual resources

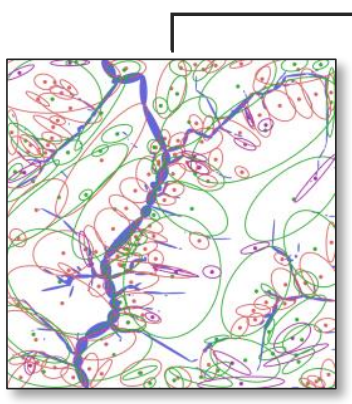

(b) Resource nodes

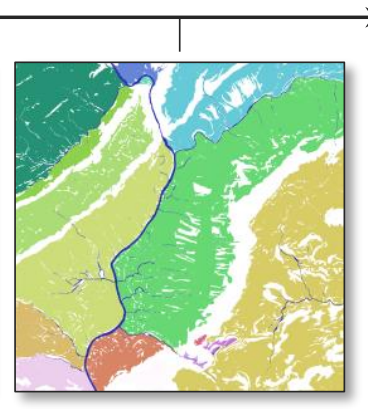

(c) Accessibility map (per species)

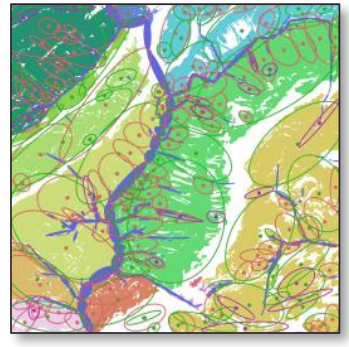

(d) Final $R A G^{i}$

Fig. 4. $R A G^{i}$ : Spatialized resources (a) are converted into species resource nodes (b), and accessibility maps are computed (c). All the nodes lying in the same colored region in (c) are finally connected by an edge labeled by the species (d). Although they span areas of arbitrary shapes, we improved salience by depicting spatialized resources as ellipses whose color, size, and location, respectively correspond to the nature, quantity, and centroid of the resource.

cross (e.g., deep water, steep slopes, overly dense vegetation, identified using species-dependent thresholds) are marked as such. We then use an iterative flood-fill algorithm to compute connected components among the remaining cells. In Fig. 4 (c), obstacles appear in white while each region is uniquely colored.

\subsection{Creating $R A G^{i+1}$ from $R A G^{i}$ and $F C L^{i}$ Density Maps}

Resource nodes and hierarchical edges. $R A G^{i+1}$ denotes a resource graph for animals. We first create water nodes (e.g., banks of a lake or a river from where animals can drink). We then use the information from $R A G^{i}$ to add a node per available localized food source among $F C L^{i}$ (either plants or other animals), as follows:

For each species $s_{j}^{i} \in F C L^{i}$, let us consider the restriction of $R A G^{i}$ to nodes where $s_{j}^{i}$ is present and the spatial edges labeled with this species. We use the density map for $s_{j}^{i}$ resulting from competition in $F C L^{i}$ (Section 4) to test the species' presence. The strongly connected components of this oriented sub-graph indicate regions where this specific $s_{j}^{i}$, now seen as a resource, is available. Therefore, we merge each strongly connected component into a single node $v \in R A G^{i+1}$ to represent this resource. The area associated with $v$ (e.g., the area covered by a plant, or the confinement region where the herd of animals resides) is extracted from $s_{j}^{i}$, s density map. To preserve the ecosystem's steady-state, we set $q(r, v)$, representing the amount of available resource, to the annual growth of $s_{j}^{i}$ in this area (computed by multiplying the number of a local specimen by the species' growth rate). Finally, hierarchical edges are created to connect $v$ to the nodes from $R A G^{i}$ used to define it.

Spatial edges. They are labeled by each species $s_{j}^{i+1}$ of animal in $F C L^{i+1}$. They are created by connecting, in both directions, all pairs of nodes (centers of ellipses in Fig. 4 (b)) that lie in the same confinement region for $s_{j}^{i+1}$, i.e., correspond to points of the same color in $s_{j}^{i+1}$, s accessibility map.
Finally, each directed edge $\left(v_{1}, v_{2}\right)$ is valued by a traveling time $t_{j}^{i}\left(v_{1}, v_{2}\right)$, where $i, j$ indicate the species, as follows: We run a shortest path algorithm on the terrain grid to calculate the best path from $v_{1}$ to $v_{2}$, taking terrain slope and the species mobility into account. The edges of the grid that exceed the species-dependent steepness threshold are removed before this computation, and their length (representing traveling time) is set from the slope. This results in curved paths that take the topography of the terrain into account. Note that both these shortest paths and their associated traveling times may differ in each direction (animal species being typically able to go down steep slopes, but not to climb them straight). The values $t_{j}^{i}\left(v_{1}, v_{2}\right)$ allow to use RAG layers for planning daily itineraries for herds. In contrast, more precise traveling speed are used when instantiating individual animals on refined paths (Section 6).

\section{ECOSYSTEM-AWARE LANDSCAPES}

\subsection{Map of Eroded Trails}

Trails are the first clue of animals' presence in a landscape, since fauna is often out of sight. Therefore, before instantiating the ecosystem in $3 \mathrm{D}$, we generate a set of trails, which are eroded according to their cumulative probability of being used. This is done by evaluating the time spent by each animal at each RAG nodes in a confinement region, assigning probabilities of fauna presence to spatial edges, and finally, refining these edges into trails.

We consider a herd $h$ of species $s$ confined in a region $C$. The probability that $h$ is present at a resource node $v$ depends on both the needs of $s$ for resources $r \in R(s)$ and the available quantities $q(r, v)$. Assuming that the animal uniformly consumes $r$ from all RAG nodes in $C$ that provide it and that the proportion of the time the herd stays on $r$ is proportional to the ratio of the annual consumption of $r$ to their total amount of necessary resources, $c(s, R(s))$, we set the probability of the presence of $s$ at $v$ to:

$$
P_{s}(v)=\sum_{r \in R(s)}\left(\frac{q(r, v)}{q(r, C)} \cdot \frac{c(s, r)}{c(s, R(s))}\right),
$$

where $q(r, C)$ is the total quantity of $r$ available in the region $C$. 
Let us now consider the herd's probability of traveling along a spatial edge $\left(v, v^{\prime}\right)$ between two RAG nodes in $C$. The approximate traveling time $t_{s}\left(v, v^{\prime}\right)$ that values this edge tells us whether the herd is likely to use this direct path or if it prefers traveling through other resource nodes. Therefore, we express the probability $P_{s}\left(v^{\prime} \mid v\right)$ for a herd at $v$ to use the spatial edge $\left(v, v^{\prime}\right)$ as the probability of going to $v^{\prime}$ weighted by the inverse of the direct traveling time (to express the fact that longer paths are less likely to be used), divided by the weighted probability to go to another resource instead:

$$
P_{s}\left(v^{\prime} \mid v\right)=\frac{t_{s}^{-1}\left(v, v^{\prime}\right) \cdot P_{s}\left(v^{\prime}\right)}{\sum_{v^{\prime \prime}}\left(t_{s}^{-1}\left(v, v^{\prime \prime}\right) \cdot P_{s}\left(v^{\prime \prime}\right)\right)} .
$$

The impact of each species $s$ on the trail's erosion in $C$ between $v$ and $v^{\prime}$ is proportional to the total mass of specimens that traveled on this spatial edge during a year. We make use of the probabilities in Eqns(1-2), considering as well the number of specimen $\|s\|$ in $C$ and their average mass $m_{s}$, to evaluate it:

$$
w_{v v^{\prime}}=\sum_{s} m_{s} \cdot\|s\|\left(P_{s}(v) \cdot P_{s}\left(v^{\prime} \mid v\right)+P_{s}\left(v^{\prime}\right) \cdot P_{s}\left(v \mid v^{\prime}\right)\right)
$$

Note that $w_{v v^{\prime}}$ stands for the cumulative weight of animals that traveled either from $v$ to $v^{\prime}$, or from $v^{\prime}$ to $v$.

Then we compute the map of eroded trails by using an undirected version of the RAG, where edges are valued by the weights $w_{v v^{\prime}}$. We initiate the eroded trails as the shortest path on the terrain corresponding to the edge of highest $w_{v v^{\prime}}$ and then iteratively extend it both on the terrain and in the RAG by adding the adjacent edges of highest weights at each end. This longest contiguous route from portions of trails, starting from the most used ones, is smoothed using spline curves. We repeat this process until all RAG edges are processed. This leads to a tree or graph structure for trails. See Fig. 5 (left), where each segment's color intensity reflects the frequency of its usage, and is set proportional to the weight. Note that the presence and reuse of several main trails often observed on natural terrains, shows the need for animals to pass through the same locations, either because the resources are abundant there or because the environment forces a passage through a specific spot. Animals scatter to the other resources from these main trails.

To make the trails visually salient during interactive exploration, we subtract them from the vegetation density maps for consistency, slightly carve the terrain based on the associated weight, and render them using muddy or rocky textures (see Figs. 1 and 5).

\subsection{Daily Planning and Instantiation of Animals in 3D}

A daily-planning for a herd $h$ of species $s$, is a sorted list Planning $(h)=$ $\left.\left.\left\{\left(v_{1}, t_{1}, t_{1}^{\prime}\right)\right), \ldots,\left(v_{n}, t_{n}, t_{n}^{\prime}\right)\right)\right\}$ of the successively visited RAG nodes of their confinement region, together the times in and out each node. Computing such plannings for herds is essential for 3D instantiation.

To evaluate how many resource nodes can be successively visited by $h$ in a single day, we use the approximate traveling times $t_{s}\left(v, v^{\prime}\right)$ on the spatial edges of the RAG and estimate the duration of stay at a given node. While in real life, the typical time a herd would spend on a resource may follow complex species-specific laws, our model lacks this specialized zoological knowledge. We instead estimate
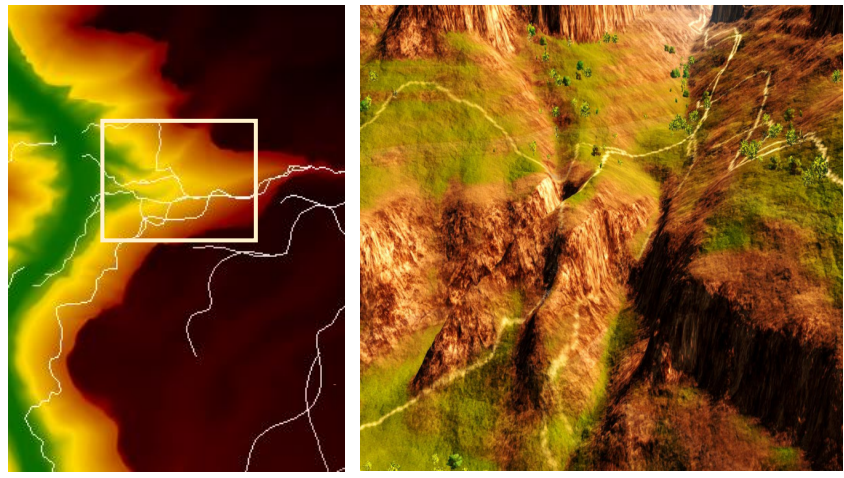

Fig. 5. Animal trails or a map (left) and the selected region during 3D exploration (right). This example, that uses the Grand Canyon DEM, shows how trails branch, going both left and right, as well as down to the valley.

the average stay time $t_{s}(v)$ according to the species' needs and the amount of resource (proportional to yearly growth) at this node.

To prevent too many animals from being at the same spot at the same time, our semi-random planning algorithm uses the concept of node capacity, defined as the maximal number of animals a node can host (in our implementation, it is proportional to the area associated to the node). We also prioritize the mandatory resources that each species needs to access every day (such as water).

The process, summarized in Algo. 3, develops as follows: At time $T=0$, we initialize Planning $(h)$ to an empty list, and set the list index $j=1$. Giving priority to mandatory resources, we randomly select a resource $r$, and the most probable node $v$ not already fully occupied by other herds where $r$ can be found, based on the probabilities $P_{s}(v)$, or on $P_{s}\left(v_{j-1} \mid v\right)$ if there was already a node $v_{j-1}$ in the list. We then decrease the capacity of $v$ by the size of the herd, update the current time to $T=t_{s}\left(v_{j-1}, v\right)$ (arrival at $v$ ) and insert $\left(v, T, T+t_{s}(v)\right)$ as node $j$ of Planning $(h)$. We update the time again to $T=T+t_{s}(v)$ (departure from $v$ ), and restart the process, until the time exceeds the period of the day when this species is awake.

Since daily plannings are solved up the food chain, the position of $v$ above is replaced, for carnivores, by the current position of the herd they are tracking, extracted from its planning. This allows for quite consistent interactions, where carnivores can be seen following herds of herbivores. See Fig. 7, top.

The computed daily-planning nodes are then used as intermediate goals for the trajectory of the herd. Path information previously computed to estimate the traveling time between resources are reused, assembled and refined using spline curves to produce a smooth result. The effective speed of each herd along their trajectory is set from the species' zoological characteristics.

During the authoring session, the result is visualized as 2D herd motion on a map, where the user clicks on resources to edit trajectories. This visualization also enables users to quickly select the areas of interest they would like to explore.

During exploration, individual animals are instantiated on the fly, depending camera position and viewing angle. They are animated using the algorithm from Reynolds [1987], which prevents collisions and generates some relative motion within the herd. Note 

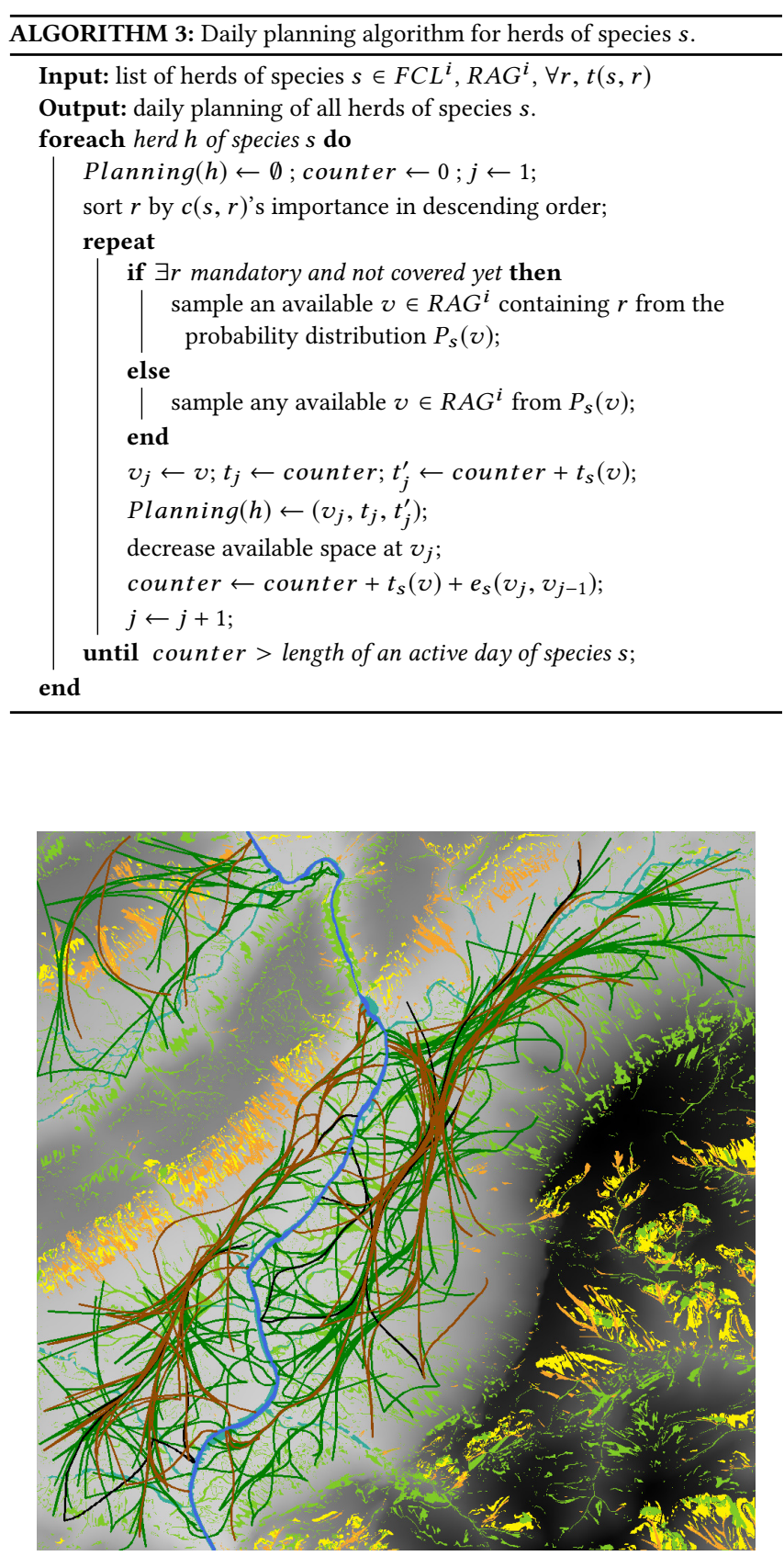

Fig. 6. Final maps, with trajectories of species in the Tautavel valley (France), including herbivores (green), wolves (black), and bears (brown). The vegetation is colored according to the main biome: mountainous (yellow), Mediterranean (orange), river-related (blue) and ubiquitous (green).

that this simple implementation, sufficient for 3D illustration in this paper's context, does not take species-specific herd shapes into account [Ecormier-Nocca et al. 2019], leaving space for improvement.
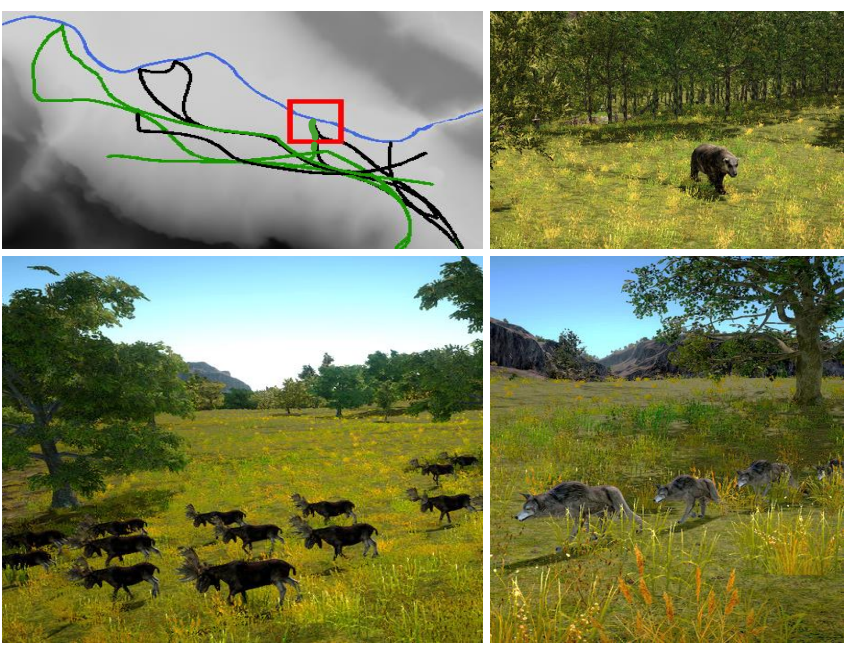

Fig. 7. Top left: Daily plannings for a herd of moose (green) and a group of wolves chasing them (black). Note that they reach the river at the same time (red square), while the wolves are chasing other herds the rest of the day. Top right and bottom: A bear, moose and wolves on their daily paths.

\section{RESULTS AND DISCUSSION}

\subsection{Implementation, Performance, and Results}

We computed all maps using Python on a desktop with an Intel Xeon E5 processor and 32GB of RAM. We ran 3D instantiation and exploration on an NVidia Quadro M2000, using Unity to allow real-time performance, although at the cost of visual realism.

We focused on medium-scale terrains of $16 \mathrm{~km}^{2}$, using e.g., $4,000 \times$ 4, 000 DEM grids with a resolution of $1 \mathrm{~m}^{2}$ per cell. The biological and zoological data is provided as supplemental material. We imported specific plant and animal models into Unity to match the different species required for the case studies.

Qualitative results. Fig. 13 shows density maps for plants and animals, where darker colors indicate higher densities. Fig. 5 depicts animal trails. Fig. 7 shows daily plannings and 3D instantiation of various animals. See the companion video for more results.

Computational time. (Table 1). Generating the full ecosystem takes on average 15 minutes, with 5 minutes for vegetation (where we use monthly temporal variations) and 5-10 minutes for the precomputation of animal paths between resources. Density maps for animals are then generated in a few seconds. Therefore, our method allows interactive editing through map over-painting, with a regeneration time ranging from a few seconds to a few minutes, depending on the stage at which the user is making changes. Once maps, textures, and daily itineraries are uploaded on Unity, the exploration runs at interactive rates.

\subsection{Validation}

The quantitative evaluation includes ablation studies to check the importance of the different parameters in our models. We also compare with previous work, and with real data and models from experts. 


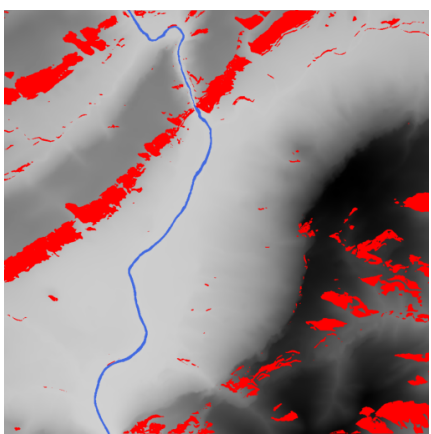

(a) Moisture independent

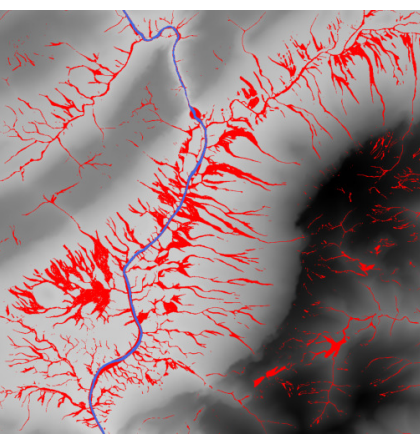

(b) Illumination independent

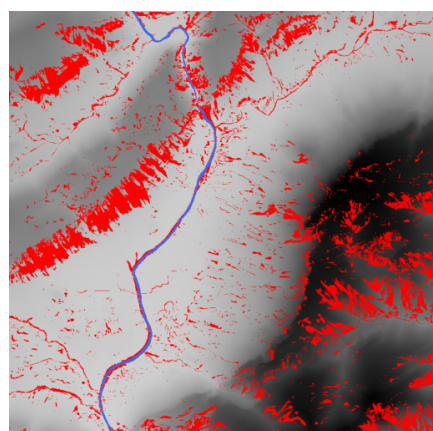

(c) Temperature independent

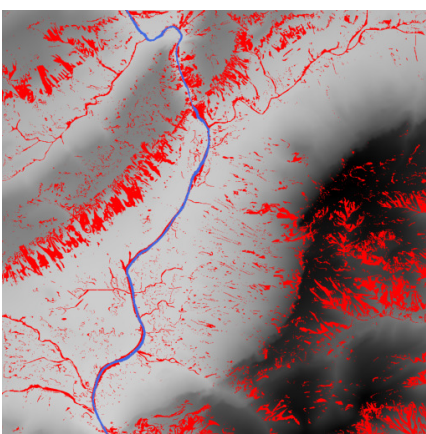

(d) Final result

Fig. 8. Ablation study for vegetation. We show the regions with the $5 \%$ highest densities of plants (all species considered), to ease comparison.

Table 1. Computational time

\begin{tabular}{l|c|c|c|c|c|c} 
Name & Cells & Species & Flora & Fauna & Trails & Total \\
\hline Mild & $4096^{2}$ & $18 / 7$ & $1400 \mathrm{~s}$ & $25 \mathrm{~s}$ & $126 \mathrm{~s}$ & $1551 \mathrm{~s}$ \\
Ice-age & $4096^{2}$ & $18 / 7$ & $1400 \mathrm{~s}$ & $24 \mathrm{~s}$ & $135 \mathrm{~s}$ & $1559 \mathrm{~s}$ \\
Grand Canyon & $3072^{2}$ & $3 / 7$ & $64 \mathrm{~s}$ & $4 \mathrm{~s}$ & $12 \mathrm{~s}$ & $76 \mathrm{~s}$ \\
Bright Angel & $390^{2}$ & $18 / 7$ & $3 \mathrm{~s}$ & $3 \mathrm{~s}$ & $2 \mathrm{~s}$ & $8 \mathrm{~s}$
\end{tabular}

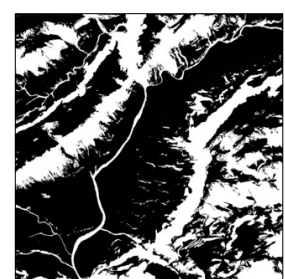

(a)

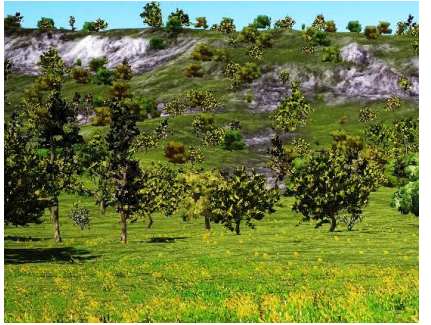

Fig. 9. Impact of animals. Top: Surplus maps (accumulated surplus of eight grass species) without (a) and with (b) fauna. The difference map (c) shows that herbivores have a strong impact in the valley and highlights trails. Bottom: Landscape without (left) and with (right) fauna.

Ablation studies. We studied the impact of plant resources by removing one resource at a time (Fig. 8). As expected, with no need for moisture (a), dense vegetation settles on well-lit, south-facing cliffs. Without any need for sun-light (b), the vegetation gets denser where moisture is abundant. Finally, without temperature bounds, the vegetation gets thicker at the top of the cliff.

We then studied the impact of fauna on the landscape (Fig. 9). Comparison of surplus maps demonstrates the impact of herbivores on their grass resource and the emergence of trails. Note that more grass resource is consumed close to the river and away from cliffs. The results demonstrate the validity and impact of individual parameters in the system for plants and animals.

Comparison with previous work. Previous work focused only on plant ecosystems, so the quantitative comparisons below are limited to vegetation. We experimented on the same terrain (extracted from the Grand-Canyon DEM data) and similar climate and species (Fig. 10). Since we did not import any preferred proportion between species in the experiment, density maps were computed using the second algorithm from Sect. 4.3. Results show that, although our procedural solution is not a simulation method, it achieves a consistent proportion of species and is quite precise in their placement (note, particularly, the denser trees at the bottom of the valley and the vegetation along stream beds).

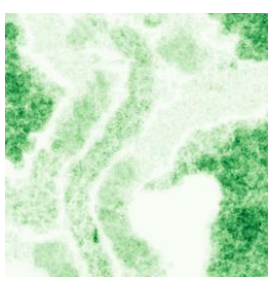

(a) Gain [2017]

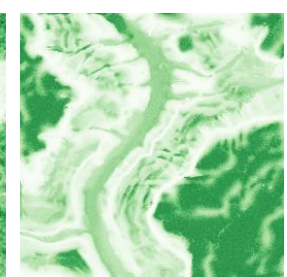

(b) Cordonnier [2017]

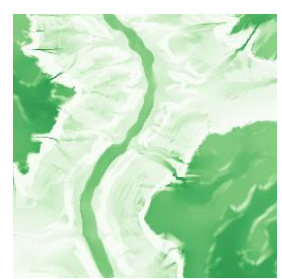

(c) Our Method

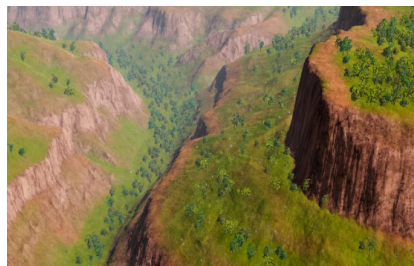

(e) Cordonnier [2017]

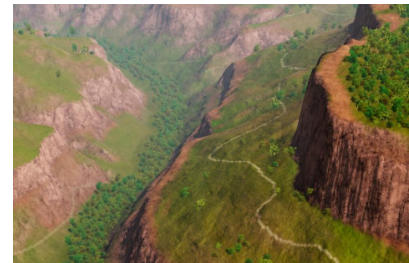

(f) Our Method
Fig. 10. Top: Vegetation density map. (a) Original result from [Gain et al. 2017], where a circular brush was used to remove some of the dense vegetation on the plateau at the bottom right. (b) Method from [Cordonnier et al. 2017] with the same environment but only three species (grass, shrub and trees). (c) Our results in similar conditions. Bottom: Resulting landscape, where our result (e) was enhanced with fauna and their trails. 
Comparison with real ecosystems. Data about the proportions of species in natural ecosystems and their localization at the terrain scale are not readily available. We confirmed this observation by contacting several professional teams of biologists. To provide a comparison with real data, we extracted from a satellite image a map of trails in a current almost wild landscape, Bright Angel valley. This enabled us to compare the trails we compute with real data. See Fig. 11. Although different, our trails look plausible, given that they are computed, in our model, to account for herds grazing activities.

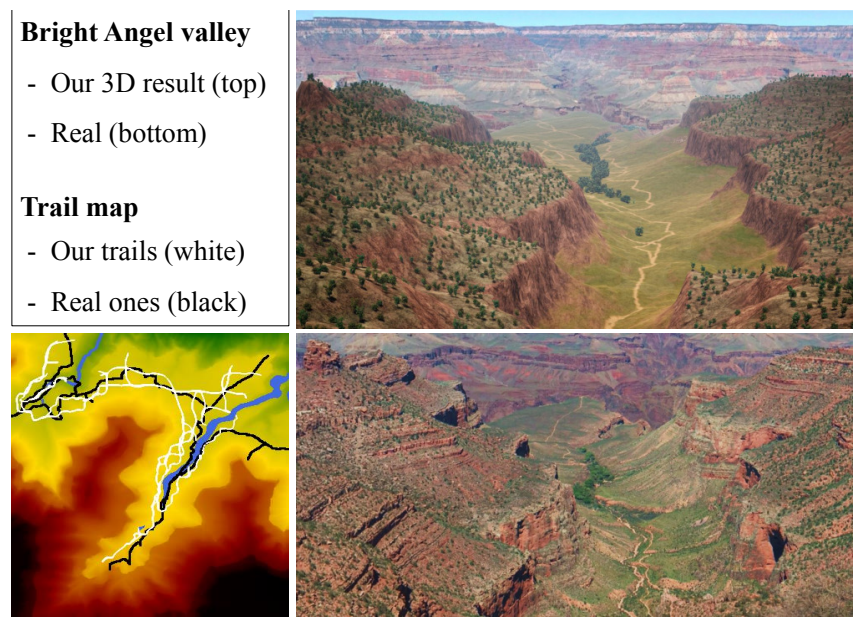

Fig. 11. Comparison with real data, using trails (left) and a photograph (right). Note that we used the real image as a background in our render.

Comparison with expert's data and models. We conducted a case study with an expert paleontologist (co-author of this paper) on the valley of Tautavel (France), studied for many years from remains (plant seeds, pollen, animal bones, etc.) extracted from a cave. Focusing on a mild, cold, and humid climate $-500,000$ years ago, favoring the development of forests, we used the expert's knowledge about species proportions for both flora and fauna as an input to our system, and then compared with the flora and fauna mobility maps the experts already produced. Note that fauna included at this period several massive species of herbivores such as bison, elk, deer, reindeer, and mouflon, as well as bear and wolf. Their mobility in the valley and on the plateau was inferred by the expert based on zoological knowledge of current, similar species. We call the resulting ecosystem mild climate in our results.

Fig. 12 compares the expert maps with our results. We grouped vegetation density maps per biome (set of species adapted to specific conditions) and rendered them as the experts did to ease comparison. We also created the same way-points in and out the valley and the same fords over the river before simulating the ecosystem's animal layers, enabling animals' access to resources to be consistent with the expert's hypotheses. The expert was impressed by the excellent match between the animals mobility results. They also stressed that when creating their flora map, three experts of different biomes worked together for four hours, while our system generated a similar looking map in a matter of minutes.
To provide a quantitative comparison between the flora maps, we computed, for each biome, the area of the intersection between the region they marked and ours, over the area of their union. This lead to the following similarity percentages: Mountainous: $67.8 \%$, Ubiquist: $69.5 \%$, River-related: $36.4 \%$, Mediterranean: $24.8 \%$.

We then discussed the main differences with the expert, leading to the following remarks: First, more river-related plant species appear in our map (blue color along stream beds), because the experts only considered the main river. Second, the Mediterranean biome (orange and striped orange/green, top-left of the maps) appears at the northwest of the plateau in their map, but at the south of the same plateau in ours. The expert interpreted this as an oversight on their end, since they did not consider the higher sun-exposure with access to water sources offered by south slopes, making them more adequate.

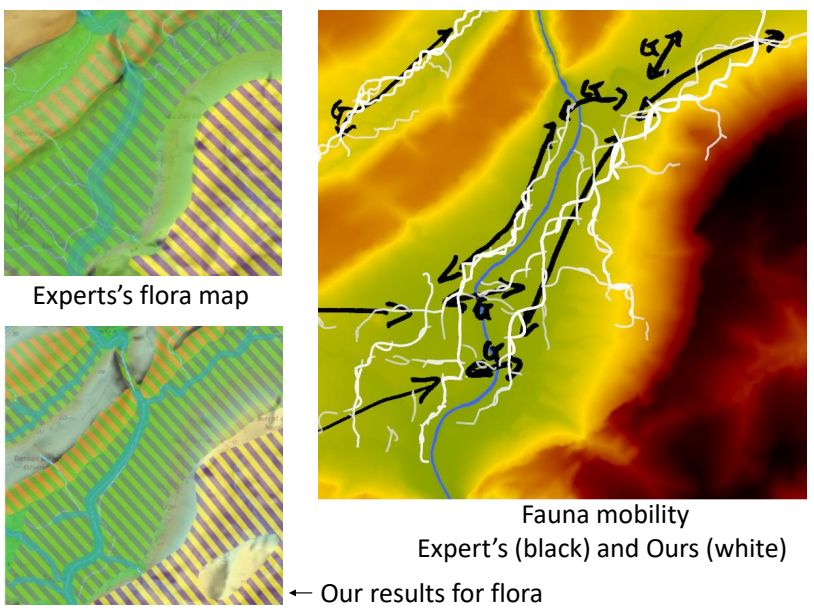

Fig. 12. Comparison with expert's maps for flora (left) and fauna (right). Left: color/stripes in blue, green, orange, and yellow stand respectively for river-related species, ubiquitous species, Mediterranean and mountainous biomes. Right: Our animal trails (white), added onto the expert's map.

Our system was then used to model an older ecosystem, which we called ice-age (around the year -550,000), corresponding to a glacial period when the freezing and dry climate was harsher to vegetation. Our density maps, based on different species proportions from the expert, are depicted in Fig. 13. They enabled the expert to visually study the difference between the way plants and animals settle in the valley under the two different climates, with a highly different spread of trees, and contrasted fauna.

\subsection{User Study}

To validate the use of our system as an authoring tool, we conducted a user study with both natural scientists and computer graphics practitioners. The first experiment was conducted with a team of nine paleontologists with expertise ranging from geology to plant biology and zoology. The second experiment was conducted with a group of ten experienced graphics users, including four professionals in animation, cinema, video games, and museography, plus five doctoral students and one intern in the field. Using the terrain 

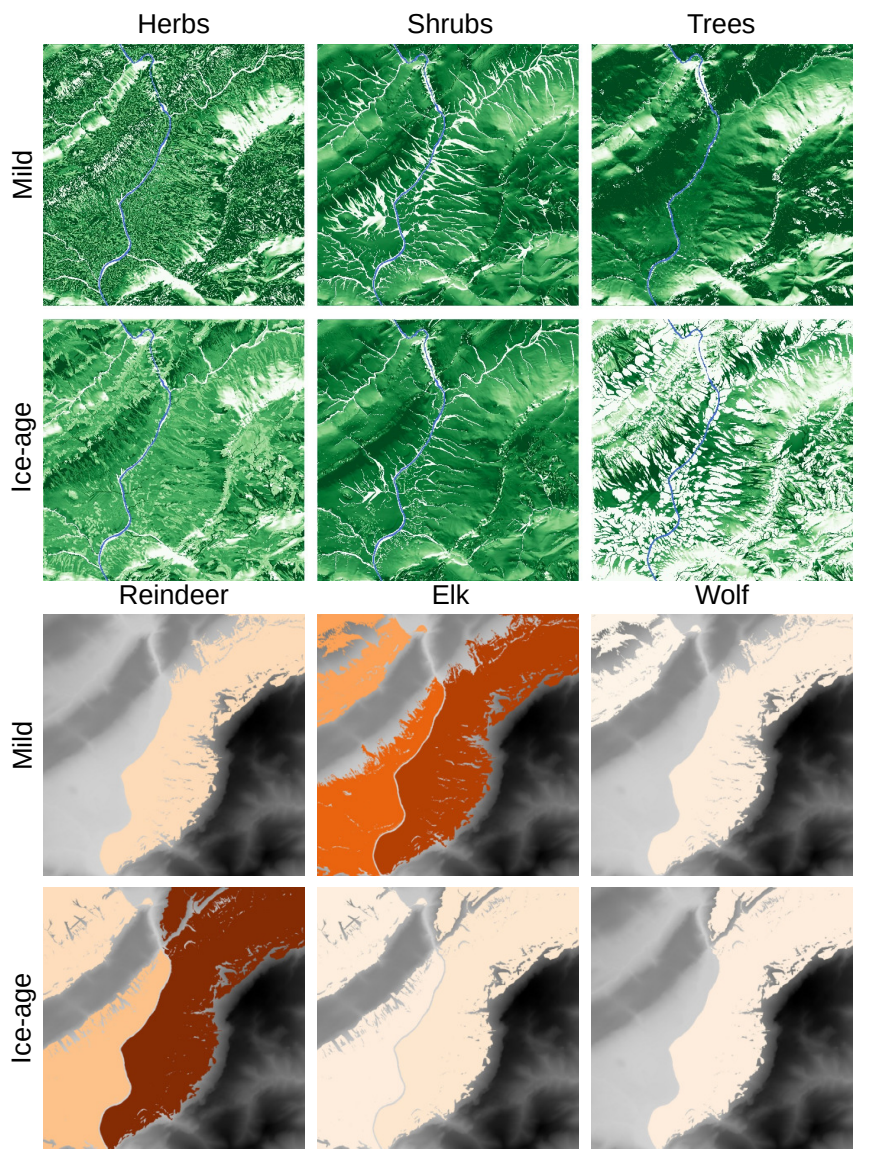

Fig. 13. Comparisons for vegetation (top) and animals (bottom), between mild climate and ice-age. The top-right map shows that a dense forest developed north of the valley in the mild climate, reducing local growth of shrubs, while fewer trees could survive in the ice-age, mostly on south slopes. The animal density maps (white to brown as density increases) show that the increased amount of reindeer (from $2 \%$ to $70 \%$ of the fauna) in ice-age allowed them to colonize a much larger region. Wolves (only $1 \%$ in the ice-age) settled on the side of the river where preys were abundant.

and set of plant species from the mild climate (enabling to use of pre-computed access maps for animals), users were allowed to input any proportion of animal species and explore the resulting maps. We briefly showed them the authoring and re-computation workflow and drove them through a five minutes exploration of the original mild landscape. Participants were then asked to mark completeness, user control, and visual realism and invited to leave any comment.

Results of the two experiments are depicted in Fig. 14. Paleontologists (upper row) were enthusiastic to use the system as an authoring and exploration tool, enabling them to check and validate their hypotheses visually. They also commented on the large saving of the expert's time, with readily available yet precise maps. On average, they rated the system's completeness as 3.9 out of 5, asking about the missing species in our prototype (indeed, we omitted including hippo, lynx, fox, and smaller animals). They rated user control as 4.1 out of 5 , being already used to paint over density maps to indicate regions. Finally, they rated realism as 4 out of 5 , mentioning that an improvement would be to develop interfaces enabling them to add more information of the fly and refine the input, such as adding more species (being used to static maps, they did not mention any lack of visual realism during 3D exploration).
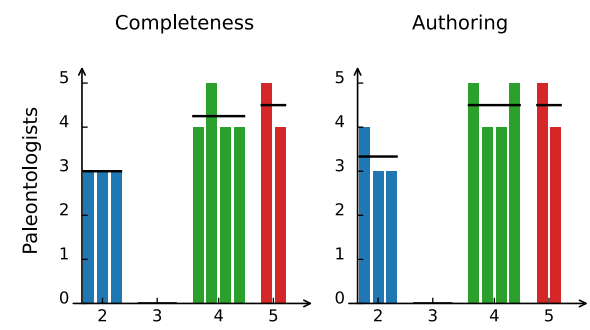

Visual realism
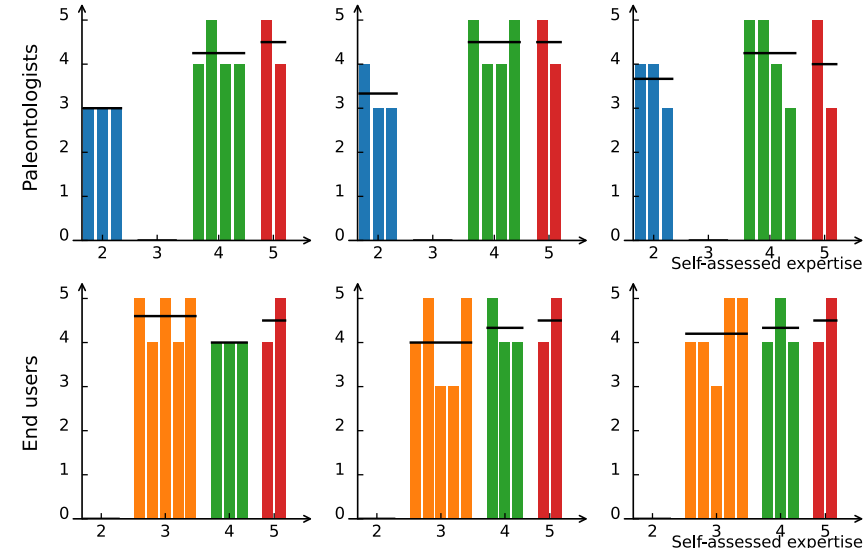

Fig. 14. Results of the user studies ran with nine experts in paleontology (top) and ten experienced graphics users (bottom), who ranged their own expertise as respectively $(2,4,5)$ and $(3,4,5)$ out of 5 .

The second experiment (bottom of Fig. 14) showed the same general trend. Since, this time, participants were CG practitioners, they were more interested in artistic authoring. They mentioned some possible improvements in user control such as enabling direct tuning of trails and animal trajectories, or the ability to control the time of the day, the season and weather conditions (our prototype always shows late morning in a sunny, early summer). Among endusers, an expert Unity practitioner commented about the time it usually takes to create animals trajectories and animation with the standard framework. While the Unity engine would help generating distributions of plants (and possibly static animals) matching the input densities, manually creating the way-points for 120 herds of 7 species would have taken at least 10 hours of tedious work.

\subsection{Limitations and Discussion}

While validation emphasized the consistency of our method and its potential to save time and effort when authoring consistent, animated landscapes, our approach still suffers several limitations.

As already stressed, our model is by construction unable to model any dynamic competition between species. Moreover, our procedural solution only approximates a steady-state ecosystem (e.g., if the surplus is fully consumed up the food chain, the species serving as a resource will be considered experiencing no natural death this given year); and realism of results may heavily depend on the user-specified proportions between species used for authoring.

Moreover, several factors for realism were neglected: Larger and more complex food chains with birds, smaller animal species, and marine resources would have to be considered for completeness. This would require to extended the method to food-chains with 
branches, which is fortunately straightforwards, since computations could still be processed by layers. The realism of the generated landscape could also be improved in many ways, e.g., modeling damage made on trees at proximity of trails taken by massive animals. More importantly, the way we model the interactions between species remains limited. Indeed, we do not handle cooperation between species [Courchamp et al. 2008], e.g., insects serving as pollinators for vegetation, or fauna's droppings helping to fertilize soil. Modeling such cooperation would create loops in the food-chain, which our current pipeline cannot handle. A possible, manual solution is for the user to re-inject some of the computed maps at a lower level of the food-chain during authoring: For instance, we re-injected a map computed as the sum of the animal densities in the ice-age of Fig. 13 as an input resource for vegetation, to show that the latter then becomes sparser in areas with little fauna. See Fig. 15. However, in turn, the new vegetation map will affect animals, and the process would need to be iterated, resulting in a dynamic ecosystem, without any theoretical guarantee of convergence.
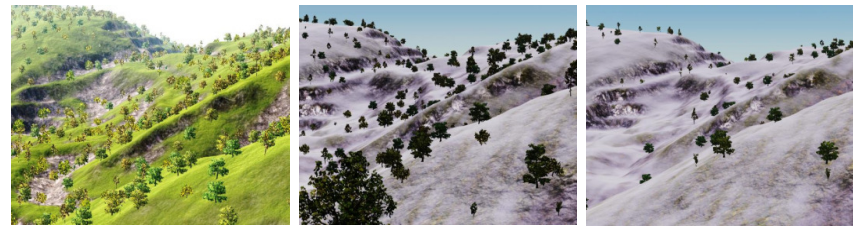

Fig. 15. Comparison between mild climate (left), ice-age (center), and ice-age where fauna fertilizes soil (loop in the food-chain) which affects vegetation.

\section{CONCLUSION AND FUTURE WORK}

We presented a method to create and instantiate a consistent, animated ecosystem over a terrain, enabling us to capture for the first time the impact of fauna on vegetation and erosion. Our method relies on a layered graph of available resources to progressively populate a steady-state ecosystem, built to match user-desired proportions. Starting with the terrain and other environmental resources, we iteratively solve competition at each food chain level. Our solution enables interactive authoring and the instantiation method allows real-time exploration of the resulting, animated landscape.

We expect designers of imaginary virtual worlds for movies or games and scientists willing to visualize and explore past, present, or future ecosystems, to share an interest in our method. In this context, enabling users to specify proportions of species not only at a given steady-state but also at specific points along a time-line would be an excellent extension for future work. The challenge would then be to create intermediate landscapes, showing the transition between two successive, predefined states. In addition to being useful for artistic story-telling, this could be a great tool for scientists, as a way to control and visualize time-evolving landscapes.

Another possible future research direction is to extend our linear food chain model to a generalized graph that would allow for a more accurate representation of the relationships between species.

Lastly, coupling our system with a standard, prey-predator simulation would bring several benefits: It would allow predicting consistent evolving ecosystems, with dynamic proportions of species, while enabling to stop at any point in time and launch a 3D exploration of the associated, animated landscape. This could be achieved by considering long-term evolution as a succession of quasi-steady states and using predicted proportions from the dynamic simulation at a given time as an input to our terrain-embedded solution.

\section{ACKNOWLEDGMENTS}

We would like to thank all the participants of the ANR SCHOPPER project for their contributions, and Gowtham Harihara for the 3D animal models. This research was funded in part by National Science Foundation grant \#10001387, Functional Proceduralization of 3D Geometric Models.

\section{REFERENCES}

M. Alsweis and O. Deussen. 2005. Modeling and Visualization of symmetric and asymmetric plant competition. In Eurographics Workshop on Natural Phenomena. Animal-Diversity-Web. DB. https://animaldiversity.org/. Accessed: 2020-01-22.

Bedrich Benes, Michel Abdul Massih, Peter Jarvis, Dadniel G. Aliaga, and Carlos A. Vanegas. 2011. Urban Ecosystem Design. In I3D. 167-174.

Guillaume Cordonnier, Eric Galin, James Gain, Bedrich Benes, Eric Guérin, Adrien Peytavie, and Marie-Paule Cani. 2017. Authoring landscapes by combining ecosystem and terrain erosion simulation. ACM Trans. Graph. 36, 4 (2017), 134.

Franck Courchamp, Ludek Berec, and Joanna Gascoigne. 2008. Allee effects in ecology and conservation. Oxford University Press.

Oliver Deussen, Pat Hanrahan, Bernd Lintermann, Radomir Měch, Matt Pharr, and Przemyslaw Prusinkiewicz. 1998. Realistic Modeling and Rendering of Plant Ecosystems. In Proc. of Sigg. (SIGGRAPH '98). ACM, 275-286.

Pierre Ecormier-Nocca, Julien Pettré, Pooran Memari, and Marie-Paule Cani. 2019. Image-based authoring of herd animations. Computer Animation and Virtual Worlds 30, 3-4 (2019).

James Gain, Harry Long, Guillaume Cordonnier, and Marie-Paule Cani. 2017. EcoBrush: Interactive Control of Visually Consistent Large-Scale Ecosystems. Eurographics 36 (2017).

Eric Galin, Eric Guérin, Adrien Peytavie, Guillaume Cordonnier, Marie-Paule Cani, Bedrich Benes, and James Gain. 2019. A Review of Digital Terrain Modeling. Computer Graphics Forum 38, 2 (2019).

Konrad Kapp, James Gain, Eric Guérin, Eric Galin, and Adrien Peytavie. 2020. Datadriven authoring of large-scale ecosystems. ACM TOG 39, 6 (2020), 1-14.

Michel Loreau. 2010. From populations to ecosystems: Theoretical foundations for a new ecological synthesis (MPB-46). Vol. 50. Princeton University Press.

Axel López, François Chaumette, Eric Marchand, and Julien Pettré. 2019. Character navigation in dynamic environments based on optical flow. Computer Graphics Forum 38, 2 (2019).

Darryl I MacKenzie, James D Nichols, J Andrew Royle, Kenneth H Pollock, Larissa Bailey, and James E Hines. 2017. Occupancy estimation and modeling: inferring patterns and dynamics of species occurrence. Elsevier.

Milosz Makowski, Torsten Hädrich, Jan Scheffczyk, Dominic L. Michels, Sören Pirk, and Wojtek Palubicki. 2019. Synthetic Silviculture: Multi-scale Modeling of Plant Ecosystems. ACM Trans. Graph. 38, 4 (2019).

Jan Ondřej, Julien Pettré, Anne-Hélène Olivier, and Stéphane Donikian. 2010. A synthetic-vision based steering approach for crowd simulation. ACM Transactions on Graphics 29, 4 (July 2010), 1.

Sébastien Paris, Julien Pettré, and Stéphane Donikian. 2007. Pedestrian Reactive Navigation for Crowd Simulation: a Predictive Approach. Computer Graphics Forum 26, 3 (Sept. 2007), 665-674. https://doi.org/10.1111/j.1467-8659.2007.01090.x

Craig W. Reynolds. 1987. Flocks, herds and schools: A distributed behavioral model ACM SIGGRAPH Computer Graphics 21, 4 (Aug. 1987), 25-34.

Craig W Reynolds. 1999. Steering Behaviors For Autonomous Characters. 21.

Stephen R Shifley, Hong S He, Heike Lischke, Wen J Wang, Wenchi Jin, Eric J Gustafson, Jonathan R Thompson, Frank R Thompson, William D Dijak, and Jian Yang. 2017. The past and future of modeling forest dynamics: from growth and yield curves to forest landscape models. Landscape ecology 32, 7 (2017), 1307-1325.

Ruben Smelik, Tim Tutenel, Rafael Bidarra, and Bedrich Benes. 2014. A Survey on Procedural Modelling for Virtual Worlds. Computer Graphics Forum 33, 6 (2014).

Tela-Botanica. DB. https://www.tela-botanica.org/. Accessed: 2020-01-22.

Jur van den Berg, Ming Lin, and Dinesh Manocha. 2008. Reciprocal Velocity Obstacles for real-time multi-agent navigation. IEEE, 1928-1935.

Xinjie Wang, Linling Zhou, Zhigang Deng, and Xiaogang Jin. 2014. Flock morphing animation. Computer Animation and Virtual Worlds 25, 3-4 (May 2014), 351-360.

Jiayi Xu, Xiaogang Jin, Yizhou Yu, Tian Shen, and Mingdong Zhou. 2008. Shapeconstrained flock animation. Computer Animation and Virtual Worlds 19, 3-4 (2008). 